\title{
Unravelling the tinnitus distress network using single trial auditory steady-state responses
}

\author{
W. Schlee ${ }^{\mathrm{a}, *}$, N. Weisz ${ }^{\mathrm{b}}$, K. Dohrmann ${ }^{\text {a }}$, T. Hartmann ${ }^{\text {a }}$, T. Elbert ${ }^{\mathrm{a}}$ \\ ${ }^{a}$ Department of Psychology, University of Konstanz, Germany \\ ${ }^{\mathrm{b}}$ Institut National de la Santé et de la Recherche Médicale, Unité 280, Lyon, France
}

\begin{abstract}
Tinnitus refers to the persistent sensation of sound in the absence of a corresponding physical source. We suggest that both the perceptual and the affective aspects of this phantom sensation arise from a distributed cerebral network whereby the functional coupling between neural nodes involved would be realised through phase-synchrony of neural oscillations. In this study, we sought to excite this network by driving it with a $37 \mathrm{~Hz}$ amplitude-modulated sound using a carrier frequency similar to the individual tinnitus ringing. Phase coherence measures between different cortical regions revealed significant differences in the excitable network between 12 tinnitus and 10 control subjects. The alterations in tinnitus were closely related to the subjective tinnitus distress ratings and resembled the patterns that have been reported for focused auditory attention. (C) 2007 Elsevier B.V. All rights reserved.
\end{abstract}

Keywords: Tinnitus; Auditory steady state response; Neural network; Phase coherence

\section{Introduction}

Tinnitus refers to the perception of ringing or buzzing in the ear in the absence of a physical sound. This sensation is most likely generated in the central nervous system as it may persist even after transsection of the auditory nerve. Correspondingly, altered cortical and subcortical functional activation and dynamics have been reported in subjects with tinnitus [1-4]. These alterations may not only reflect mechanisms related to the tinnitus sensation but also processes that result in affective colouring and directed attention towards the percept. The result, it seems, must be a self-regulating tinnitus-related neural network. Phase synchronization in distinct frequency bands may be a signature of such a distributed network of neural nodes, as it may indicate cooperation between distinct cell assemblies [5].

* Corresponding author. Tel.: +49 7531 884612; fax: +49 7531882891.

E-mail address: winfried.schlee@uni-konstanz.de (W. Schlee). 
Such synchronization might also be implied in a neural tinnitus-distress network. Since tinnitus is a persistent sensation, the regions involved in tinnitus processing are probably activated in sync. Given Hebbian learning, this should lead to a strengthening of the connections between activated cell assemblies and thus to a formation of an ever-stronger neuronal network.

In this study we wanted to probe such a network and scrutinize its architecture by driving involved brain regions with a $37-\mathrm{Hz}$ amplitude modulated sound. Stimulation with a sound that bears some similarity with the tinnitus percept should evoke the tinnitus network, while stimulation with a control sound, unrelated to tinnitus characteristics, should not. We used MEG to monitor synchronized brain activity by means of phase coherence of evoked responses resulting from different brain regions.

\section{Materials and methods}

Subjects: Twelve individuals with chronic tinnitus (seven women; mean age \pm sd: $27.9 \pm$ 8.6, mean tinnitus duration in years $\pm \mathrm{sd}$ : $5.8 \pm 4.2$ ) and 10 normal hearing controls (five women; mean age \pm sd $25.7 \pm 2.7$ ) participated in the study. All participants were righthanded according to the Edinburgh Handedness Inventory [6].

Design and stimulation: Thirty trials of $37-\mathrm{Hz}$ amplitude modulated tones with a duration of $10 \mathrm{~s}$ each were presented in each condition. The tinnitus-related sound frequency was selected according to the individual subjective tinnitus spectrum [7], taking into account dead regions (inner hair cell loss) on the cochlea [8]. The front edge of the dead region and the plateau of the tinnitus spectrum often coincide [9] and this point was chosen as the tinnitus-related tone. Two tones with their carrier frequencies 1.1 and 2.2 octaves below were used as control tones.

Data acquisition: MEG recordings were carried out using a 148-channel magnetometer system (MAGNES 2500 WH, 4D Neuroimaging, San Diego, USA), installed in a magnetically shielded room (Vakuumschmelze Hanau). The subjects were instructed to lie quietly in a comfortable supine position. The data was recorded with a sampling rate of $678.17 \mathrm{~Hz}$ and a hard-wired high-pass filter of $0.1 \mathrm{~Hz}$.

Data pre-processing: The signals were projected to a source montage of eight regional dipoles using BESA. The same source montage was used for all subjects and was adjusted to the individual head size. It consisted of eight regional sources: two in the temporal plane (left and right) approximately at the location of Heschl's gyrus, two in the prefrontal area (left and right), two in the parietal lobe (left and right), one in the posterior region (midline), and one frontocentral source located nearly at the anterior cingulum. A bandpass filter (35 to $39 \mathrm{~Hz}$ ) was applied to the data; we segmented the data in windows of $148.32 \mathrm{~ms}$ and averaged them in the time domain. Only phase data of the $37-\mathrm{Hz}$ response were used for the calculation of inter-regional synchrony. This value was calculated from the complex values returned by a Fast Fourier Transformation of the time series.

Data analysis: Using the thirty trials of each condition, we calculated a phase locking value (PLV) [10] for all possible connections between the eight regions. A mixed-model ANOVA revealed differences in the between-regions PLV between tinnitus subjects and controls. Values for these connectivities were correlated with the subjective tinnitus distress ratings, as assessed by the German version of the Tinnitus Questionnaire [11]. 

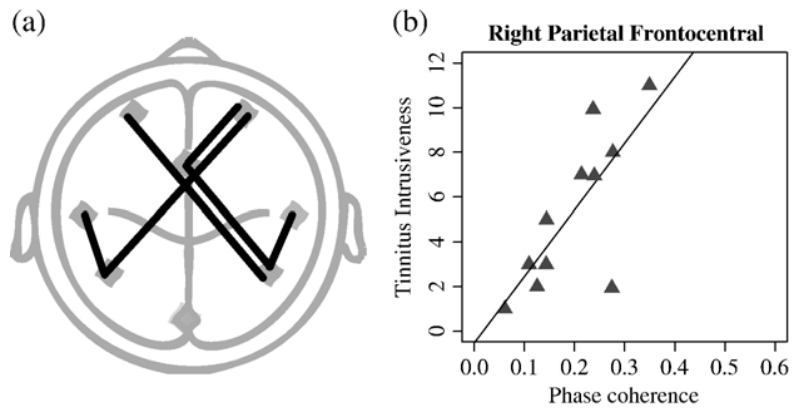

Fig. 1. Connected pairs that reveal significant group difference in phase coherence as well as strong correlations between PLV and the subjective tinnitus rating (a). Scatterplot showing a close relationship between the subjective rating of tinnitus intrusiveness (Tinnitus Questionnaire) and the phase coherence between right parietal and frontocentral sources (b).

Filtering and averaging were carried out using EEGLAB (http://www.sccn.ucsd.edu/ eeglab) and MATLAB (Mathworks, Version 6.5, Release 13), respectively. For the statistical analysis we used R (http://www.r-project.org, R Development Core Team, 2005).

\section{Results}

The analysis of variance revealed various connectivities with differences between the tinnitus and the control group. The PLV measured between some of these connected pairs showed moderate to strong correlations with the subjective tinnitus ratings when the auditory stimulation was conducted with a carrier frequency similar to the individual tinnitus tone. They are depicted in Fig. 1(a). The strongest, a near-perfect relationship, is illustrated in the scatterplot in Fig. 1(b); the other correlations are listed in Table 1. There was no or little statistical support for associations between the tinnitus ratings and the PLV in the control conditions.

\section{Discussion}

In this work we demonstrate the existence of a tinnitus-related cortical network. Furthermore, several coherent activations were found to be highly correlated with the subjective

Table 1

Significant relationships between subjective tinnitus ratings and phase coherence measures

\begin{tabular}{lllr}
\hline TQ subscale & Phase coherence & Pearson's $r$ & $p$ \\
\hline Tinnitus Intrusiveness & Frontocentral-right frontal & -0.65 & 0.029 \\
Tinnitus Intrusiveness & Left parietal-right frontal & -0.64 & 0.035 \\
Tinnitus Intrusiveness & Frontocentral-right parietal & +0.76 & $<0.01$ \\
Tinnitus Intrusiveness & Right temporal-right parietal & +0.74 & $<0.01$ \\
Emotional Distress & Left temporal-left parietal & -0.63 & 0.036 \\
Emotional Distress & Left parietal-right frontal & -0.61 & 0.049 \\
Emotional Distress & Frontocentral-right parietal & +0.71 & 0.013 \\
Emotional Distress & Left frontal-right parietal & -0.61 & 0.049 \\
Cognitive Distress & Left frontal-right parietal & -0.73 & 0.010 \\
\hline
\end{tabular}


ratings of tinnitus distress. Thus, we found support for the hypothesis that synchronized brain oscillations are the neural basis for the perception and affective colouring of the tinnitus sound. The current data suggest that we need to consider a wide-spread network rather than a single activation spot to understand the mechanisms of tinnitus generation.

The strong correlations between the subjective tinnitus distress ratings and the objective measure of the phase coherence in the MEG data provide confidence in the functional connectivity that exists between them. Conclusions about the exact mechanism on tinnitus generators, however, are not possible based on this correlational data set. It cannot be decided whether the synchronizations observed here are responsible for the generation of the tinnitus sound or whether they are a consequence of the phantom sensation reflecting more the distress that is associated with it.

Conclusions about the exact architecture of the network cannot be drawn from this data. Future research must combine structural data and MEG data to unravel the brain structures involved. However, the available data may already allow us to model the observed pattern of network activation. With the prominent role of the right parietal cortex, the temporal regions and the anterior cingulum, this network closely resembles the PET-covariations that Zatorre [12] found in tasks of auditory attention. This supports the idea of a strong attentional component in the tinnitus processing as stated by other authors $[13,14]$.

\section{Acknowledgements}

This work was supported by the DFG (EL, 101/20-5) and the Tinnitus Research Initiative (TE0602).

\section{References}

[1] A.H. Lockwood, et al., The functional neuroanatomy of tinnitus: evidence for limbic system links and neural plasticity, Neurology 50 (1) (1998) 114-120.

[2] F. Mirz, et al., Positron emission tomography of cortical centers of tinnitus, Hear. Res. 134 (1-2) (1999) $133-144$.

[3] M. Muhlau, et al., Structural brain changes in tinnitus, Cereb. Cortex 16 (9) (2006) 1283-1288.

[4] N. Weisz, et al., Tinnitus perception and distress is related to abnormal spontaneous brain activity as measured by magnetoencephalography, PLoS Med. 2 (6) (2005) e153.

[5] T. Elbert, A. Keil, Imaging in the fourth dimension, Nature 404 (6773) (2000) 29-31.

[6] R.C. Oldfield, The assessment and analysis of handedness: the Edinburgh inventory, Neuropsychologia 9 (1) (1971) 97-113.

[7] A. Norena, et al., Psychoacoustic characterization of the tinnitus spectrum: implications for the underlying mechanisms of tinnitus, Audiol. Neuro-Otol. 7 (6) (2002) 358-369.

[8] B.C. Moore, et al., A test for the diagnosis of dead regions in the cochlea, Br. J. Audiol. 34 (4) (2000) $205-224$.

[9] N. Weisz, T. Hartmann, K. Dohrmann, W. Schlee, A. Norena, High-frequency tinnitus without hearing loss does not mean absence of deafferentation, Hear. Res. 222 (1-2) (2006) 108-114.

[10] J.P. Lachaux, et al., Measuring phase synchrony in brain signals, Hum. Brain Mapp. 8 (4) (1999) 194-208.

[11] G. Goebel, W. Hiller, Tinnitus-Fragebogen (TF): Ein Instrument zur Erfassung von Belastung und Schweregrad bei Tinnitus, Hogrefe, Göttingen, 1998.

[12] R.J. Zatorre, T.A. Mondor, A.C. Evans, Auditory attention to space and frequency activates similar cerebral systems, NeuroImage 10 (5) (1999) 544-554.

[13] R. Hallam, Psychological approaches to the evaluation and management of tinnitus distress, in: J. Hazell (Ed.), Tinnitus, Churchill and Livingston, London, 1986, pp. 1-50.

[14] G.P. Jacobson, et al., Electrophysiological indices of selective auditory attention in subjects with and without tinnitus, Hear. Res. 97 (1-2) (1996) 66-74. 